\title{
An algorithm for solving the Burgers-Huxley equation using the Elzaki transform
}

\author{
Adedapo Chris Loyinmi ${ }^{1}$ (D) Timilehin Kingsley Akinfe ${ }^{1}$ (I)
}

Received: 8 October 2019 / Accepted: 8 November 2019 / Published online: 2 December 2019

(c) Springer Nature Switzerland AG 2019

\begin{abstract}
In this research work, we propose an algorithm which involves the coupling of a new integral transform called the Elzaki transform and the well-known homotopy perturbation method on the Burgers-Huxley equation which is a type of nonlinear advection-diffusion partial differential equation. The Burgers-Huxley equation which models reaction mechanisms, diffusion transports and nerve ion propagation which is applicable in traffic flows, acoustics, turbulence theory, hydrodynamics and generally mechanics is the fusion of the well-known Burgers equation and the Huxley equation. We proffer an analytical solution in the form of a Taylor multivariate series of displacement $x$ and time $t$ using the proposed Elzaki homotopy transformation perturbation method (EHTPM) to three cases of the Burgers-Huxley equation. This solution converges rapidly to a closed form which is the same as the exact solutions obtained using the normal analytical methods from the existing literatures. The exact results and that of our proposed EHTPM when compared via tables and 3D plots show an excellent agreement devoid of errors.
\end{abstract}

Keywords Nonlinear partial differential equations (PDE) · Advection-diffusion equation · Burgers-Huxley equation . Elzaki transform $\cdot$ Homotopy perturbation method $\cdot$ Acoustics

Mathematics Subject Classification $35 \mathrm{~A} 22 \cdot 35 \mathrm{~A} 35 \cdot 35 \mathrm{~N} 20 \cdot 65 \mathrm{R} 10 \cdot 35 \mathrm{Q} 35 \cdot 35 \mathrm{~K} 55 \cdot 35 \mathrm{~K} 57 \cdot 65 \mathrm{~A} 05 \cdot 65 \mathrm{H} 20$

\section{Introduction}

A partial differential equation (PDE) is an equation involving functions of more than one independent variable and their partial derivatives. They occur in many applications and play a big role in engineering and applied sciences [1]. For instance, a second-order partial differential equation for the function $u(x, y)$ is $F\left(x, y, u, u_{x}, u_{y}, u_{x x}, u_{y y}, u_{x y}\right)=0$ where the function $F$ is given. An equation is said to be linear if the unknown function and its derivatives are linear in $F$.

An example of a first-order linear equation is

$a(x, y) u_{x}+b(x, y) u_{y}+c(x, y) u=f(x, y)$ where the functions $a, b, c$ and $f$ are given. Contrary to this, the equation is nonlinear. Nonlinear equations are equations with nonlinear terms.

Nonlinear partial differential equation (NPDE) has been widely studied by numerous researchers over the years and has become ubiquitous in nature [2-8]; it can be classified into integrable and non-integrable.

The integrable equations are those whose behaviour is determined by their initial conditions and, as a matter of fact, can be solved by integrating them from those initial conditions. However, some nonlinear PDEs are integrable after some symbolic transformation. These equations have numerous exact solutions being constructed to them

Adedapo Chris Loyinmi, loyinmiac@tasued.edu.ng; Timilehin Kingsley Akinfe, akinfetimilehin@gmail.com | ${ }^{1}$ Department of Mathematics, Tai Solarin University of Education, ljagun, ljebu Ode, Ogun State, Nigeria. 
but, however, still need the attention of mathematicians as there is no single best method for a model/problem.

The preference of a method to another lies in the rapid convergence to exact results, computational stress and simplicity of the method. However, some methods can perform better on some models than others due to the nonlinearity of the model and the radius of convergence of such method. Necdet Bildik and some other authors buttressed this based on their convergence analysis on some iterative methods $[9,10]$.

Amongst these integrable equations are the Benjamin-Ono equation relevant in internal waves in deep water, nonlinear Schrödinger equation applicable in optics and water waves, Kadomtsev-Petviashvili equation applied in shallow water waves, Korteweg-de Vries $(\mathrm{KdV})$ equation applicable in shallow waves models, the sine-Gordon equation applicable in solitions and quantum field theory and so on [11].

On the contrary, non-integrable equations, viz. the Ginzburg-Landau equation applied in the theory of conductivity, Fisher's equation applied in genetic propagation, Burgers-Huxley equation applicable in advection-diffusion models and so on, require special attention, methods and constructive algorithm in order to obtain their exact solution. They are known to have few or no exact solutions.

However, just like the integrable equations, there are some numerous remarkable methods which are used to obtain exact and explicit solutions of non-integrable PDEs. The most remarkable ones are the Jacobi elliptic function method, the tanh function method, Weierstrass elliptic function method, Hirota bilinear method [12] to mention a few.

Exact solution rarely exists generally for nonlinear differential equations (ordinary and partial), and as a result of this, efforts have been made over the years in constructing semi-analytical and numerical means or schemes/algorithms for solving them, viz. Taylor collocation method [13, 14], Euler collocation methods [15], wavelet collocation methods $[5,6,16]$, iterative differential quadrature method (IDQ) [17], variational iteration method [7, 18, 19], homotopy perturbation method [20-23], perturbation iteration method [10, 24], Adomian decomposition method [9], new iterative method [25] and homotopy analysis method [26, 27].

The investigation and development of travelling wave solution play a prominent role in nonlinear science, and numerous soliton scientists have also endeavoured to seek the solitary wave solution of numerous models including the Burgers-Huxley equation [28-31].

In quest of seeking exact solutions for model/equations, mathematicians have also come up with hybrid methods (coupling two distinct methods) to obtain exact solutions of some models, viz. Sumudu decomposition method
[32-34], homotopy perturbation transformation method $[35,36]$, homotopy variational iteration method [37], Elzaki differential transform [38], Elzaki projected differential transform [39, 40], Elzaki homotopy transformation perturbation method [41-43], Laplace Adomian decomposition method [44] and Laplace variational iteration method [45-47].

Very recently, Ziane et al. have coupled Elzaki integral transform and variational iteration method on partial differential equations of fractional order [48]; Dhunde and Waghmare [4] have coupled the double Laplace transform with the new iterative method on nonlinear partial differential equations; Jena and Chakraverty [43] have coupled the Elzaki transform with the homotopy perturbation on time-fractional Navier-Stokes equation of which a series solution which converges rapidly to the exact solutions of the problems was obtained using these proposed methods.

In this paper, we applied an unprecedented hybrid method that involves a new integral transform (Elzaki transform) which is a modification of Sumudu integral transform with the well-known homotopy perturbation method on the Burgers-Huxley equation of three cases as a result of variation of equation parameters. The solution of these three cases is found to be an infinite multivariate Taylor series which rapidly converges to the exact solution of the problem.

The Elzaki transform was invented by Elzaki [49] and was derived from the classical Fourier integral and a modification of the existing Sumudu transform. Based on the mathematical simplicity of the Elzaki transform, it facilitates the process of solving ordinary and partial differential equations in the time domain [50-52]. The transform is defined by

$T(v)=v \int_{0}^{\infty} f(t) \mathrm{e}^{-\frac{t}{v}} \mathrm{~d} t$

Here, $f(t)$ is a function of time.

The idea of homotopy perturbation (HPM) [53] was introduced by Ji-Huan $\mathrm{He}$, who merged the traditional perturbation technique with the homotopy in topology by constructing a convex homotopy, considering the unknown function to a be an infinite series of an imbedding parameter $p \in[0,1]$ (perturbing the unknown function), and then obtain the solution of the problem in series form converging to the exact solution. He used it to solve strongly nonlinear problems in applied sciences, such as the Duffing equations and the eardrum equations [20-22, 53].

Unlike nonlinear PDEs, linear partial differential equations could be solved analytically and by using integral transforms like the Elzaki transform [50], Laplace, Sumudu 
$[54,55]$ and Fourier transforms. But due to the complexity caused by the nonlinear terms, these integral transforms are totally incapable of solving nonlinear partial differential equations. In this case, we introduce a semi-analytical method (homotopy perturbation method) so as to handle the nonlinear terms (Fig. 1).

In addition, the Elzaki transform is an analytical method which provides exact solutions, while the homotopy perturbation is a semi-analytical method which provides approximate solutions. As a result, coupling these two methods would definitely yield a result extremely similar and highly convergent to the exact solution of the problem.

The huge advantage of this algorithm using Elzaki transform lies in the capability of combining two powerful methods with the need of only initial conditions for obtaining an exact solution of the nonlinear advection-diffusion equation (Fig. 2).

\section{The Burgers-Huxley equation}

The Burgers-Huxley equation is a nonlinear partial differential equation which describes a wide class of physical nonlinear phenomena. It describes the interaction between reaction mechanisms, convection effects and

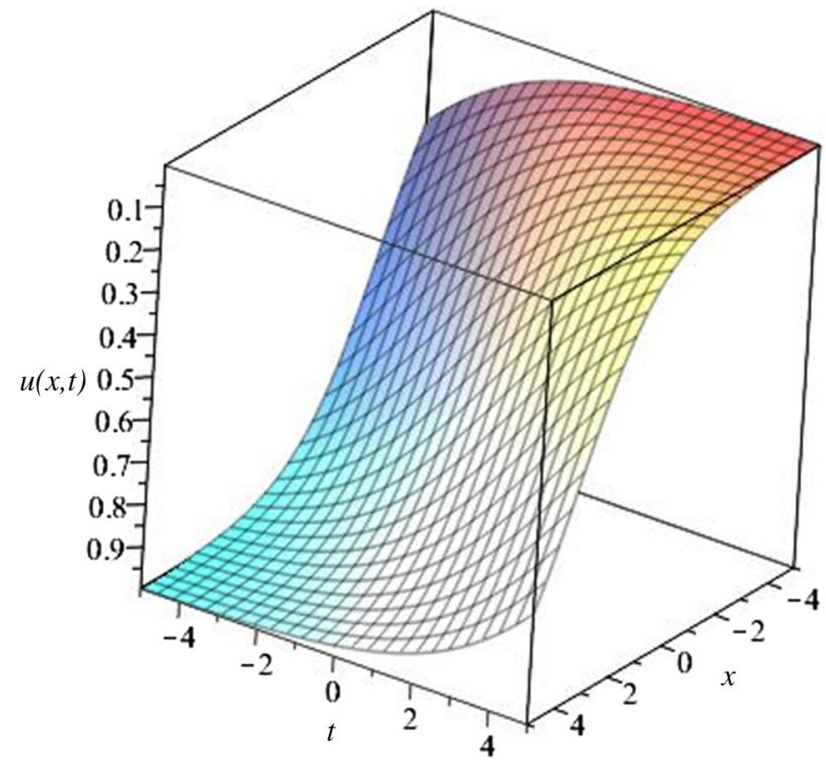

Fig. 1 Solution plots for the closed form in Eq. (47) and the travelling wave solution in Eq. (48) for $\alpha=0, \delta=1, \gamma=1$ and $\beta=1$ (Case 1)

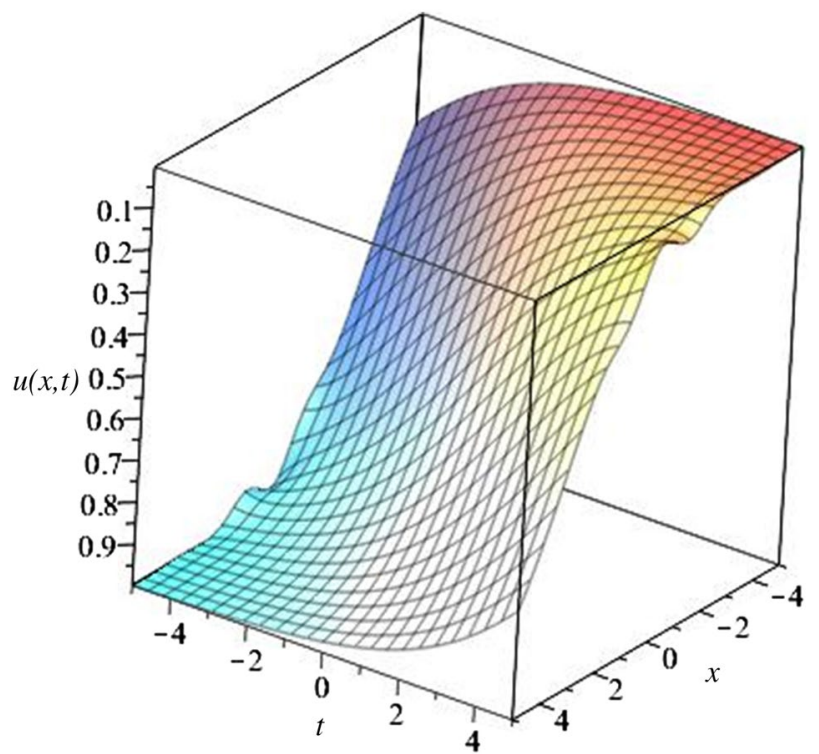

Fig. 2 Solution plots of the multivariate series obtained in Eq. (45) using the proposed Elzaki homotopy transformation perturbation method (EHTPM) (Case 1)

diffusion transports. It finds its application in many fields such as biology, nonlinear acoustics, metallurgy, chemistry, combustion, mathematics and engineering by Satsuma et al. [56]. The Burgers-Huxley equation came into existence due to the combined efforts of Bateman [57,58], Burgers [59] for the Burgers equation and also Hodgkin and Huxley [60] for the Huxley equation.

The generalized form of the Burgers-Huxley equation is given as (Fig. 3):

$u_{t}=u_{x x}-\alpha u^{\delta} u_{x}+\beta u\left(1-u^{\delta}\right)\left(u^{\delta}-\gamma\right)$

$0 \leq x \leq 1, t \geq 0$; with the initial condition of Eq. (2) is subjected to

$u(x, 0)=\left[\frac{\gamma}{2}+\frac{\gamma}{2} \tanh (\sigma \gamma x)\right]^{\frac{1}{\delta}}$

where

$\sigma=\frac{\delta\left(\sqrt{\alpha^{2}+4 \beta(1+\delta)-\alpha}\right)}{4(1+\delta)}$

$\beta \geq 0$ is the reaction coefficient, $\alpha>0$ is the advection coefficient, $\gamma$ and $\delta$ are real constants with $\gamma \in(0,1)$ and $\delta>0, u_{x x}$ is the diffusive term, $u^{\delta} u_{x}$ is the advection term, while $u\left(1-u^{\delta}\right)\left(u^{\delta}-\gamma\right)$ is the reaction term. 


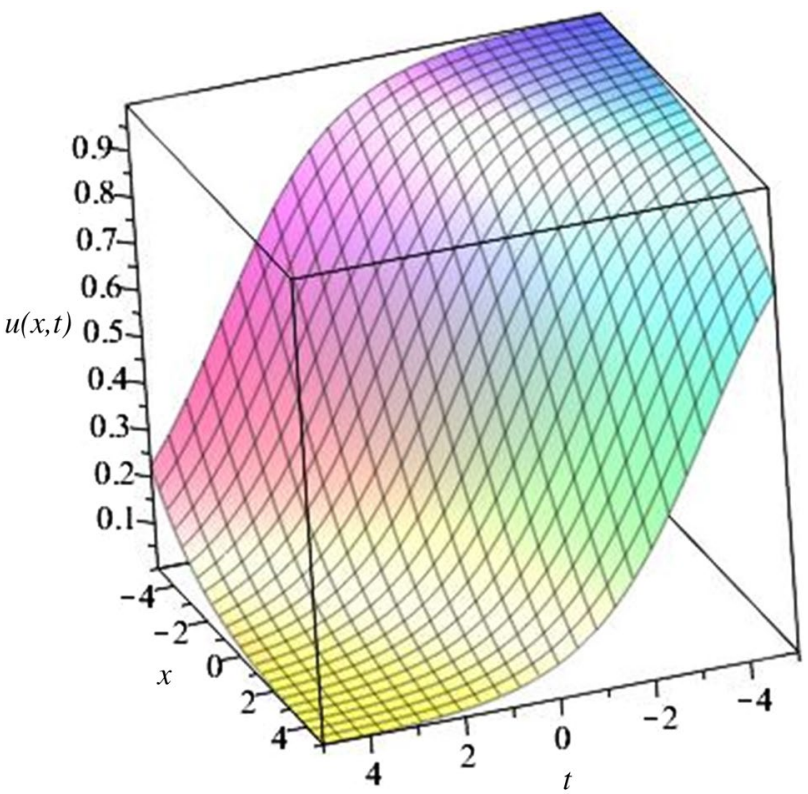

Fig. 3 Solution plots for the closed form in Eq. (65) and the travelling wave solution in Eq. (66) for $\alpha=-1, \delta=1, \gamma=1$ and $\beta=1$ (Case 2)

The above nonlinear partial differential Eq. (2) models the interaction between reaction mechanisms, convection effects and diffusion transports [56].

When $\alpha=0$ and $\delta=1$, then Eq. (2) is reduced to the 'Huxley's equation'. This describes the nerve propagation in nerve fibres and wall motion in liquid crystals [28].

When $\beta=0$ and $\delta=1$, Eq. (2) reduces to'Burgers equation'. This describes the far field of wave propagation in nonlinear dissipative system [58].

Several attempts have been made by researchers to obtain the exact or analytical and numerical solution of the Burgers-Huxley equation over time.

An analytical solution was proffered to a fractional Burgers-Huxley equation using the residual power series method by Freihat [61], and an exact solution and special form of the Burgers-Huxley equation were obtained using the $\left(G^{\prime} / G\right)$ expansion method by Zhu [62]. Nourazar et al. [63] obtained the exact solution of the Burgers-Huxley equation using the homotopy perturbation method, Mittal and Tripathi [64] obtained numerical solutions of the generalized Burgers-Fisher and generalized Burgers-Huxley equations using the collocation of cubic B-splines, Kamboj and Sharma [65] through iteration obtained an analytical solution to a singularly perturbed Burgers-Huxley equation, and Feng et al. [66] obtained a travelling wave solution to the Burgers-Huxley equation (Fig. 4).

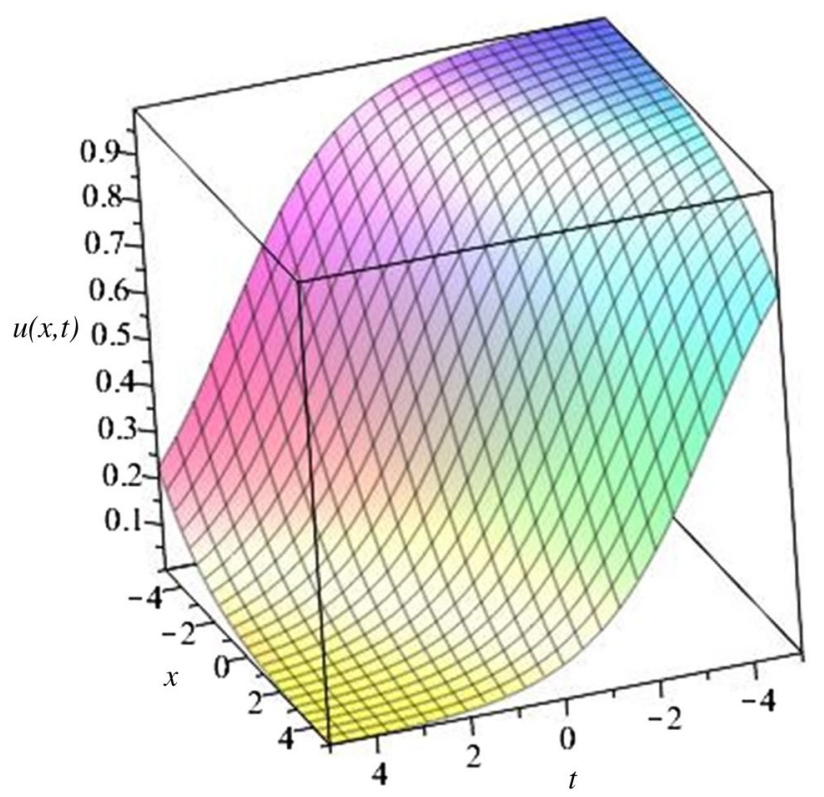

Fig. 4 Solution plots of the multivariate series obtained in Eq. (63) using the proposed Elzaki homotopy transformation perturbation method (EHTPM) (Case 2)

A solitary wave solution was obtained for the generalized Burgers-Huxley equation by El-Danaf [29], while Tomasiello [17] obtained a numerical solution of the Burgers-Huxley equation using the iterative differential quadrature (IDQ) method (Fig. 5).

Furthermore, a travelling wave solution of the generalized Burgers-Huxley equation was obtained by Deng [67], while Hashim et al. [9] solved the generalized Burgers-Huxley equation using the Adomian decomposition method and so on (Fig. 6).

\section{Elzaki transform}

The Elzaki transform is a semi-infinite convergent integral of the form.

$T(v)=v \int_{0}^{\infty} f(t) \mathrm{e}^{-\frac{t}{v}} \mathrm{~d} t$

Or

$T(v)=v^{2} \int_{0}^{\infty} f(v t) \mathrm{e}^{-t} \mathrm{~d} t$ 


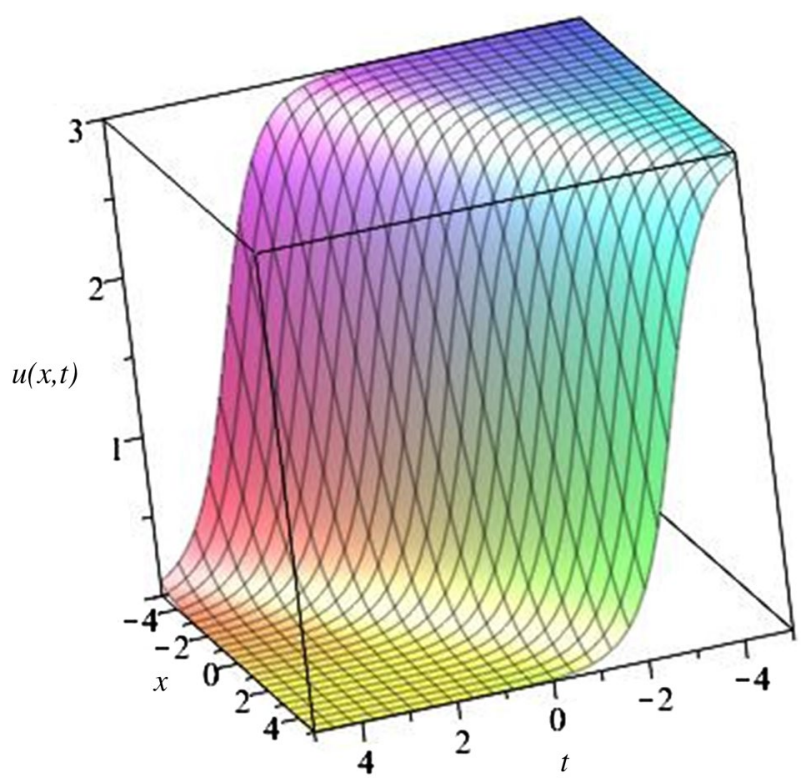

Fig. 5 Solution plots for the closed form in Eq. (82) and the travelling wave solution in Eq. (83) for $\alpha=-2, \delta=1, \gamma=3$ and $\beta=1$ (Case 3)

The function $f$ is of exponential order in the set $A=\left\{f(t): \ni m, k_{1}, k_{2},>0,|f(t)|<M e^{\frac{|t|}{k_{j}}}\right.$

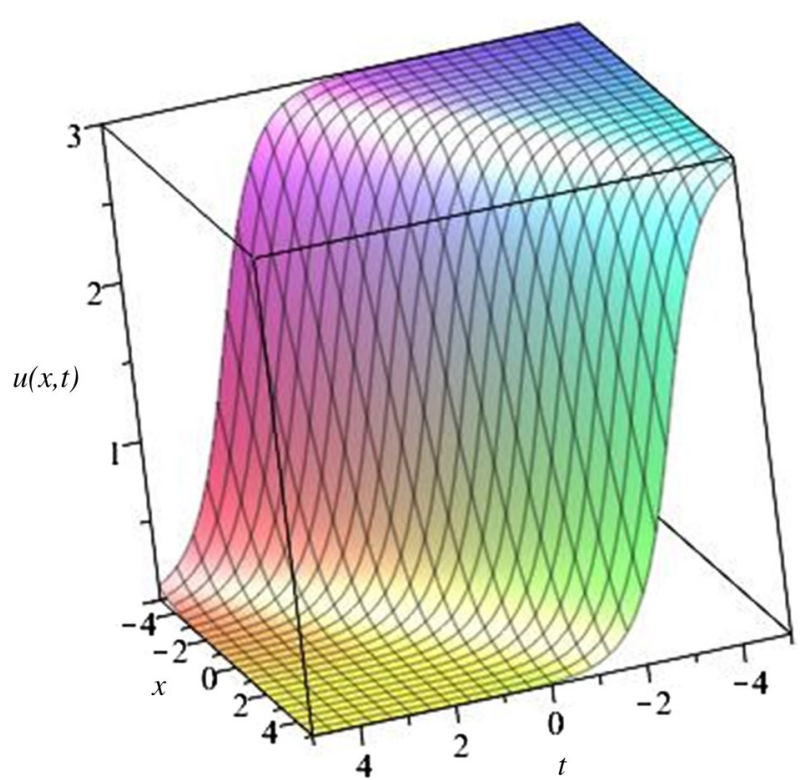

Fig. 6 Solution plots of the multivariate series obtained in Eq. (80) using the proposed Elzaki homotopy transformation perturbation method (EHTPM) (Case 3)
If

$\left.t \in(-1)^{j} X[0, \infty]\right\}$

then

$E\{f(t)\}=T(v)=v \int_{0}^{\infty} f(t) \mathrm{e}^{-\frac{t}{v}} \mathrm{~d} t$

It is called modified Sumudu transform invented/introduced by Tarig M. Elzaki.

By proceeding, we have the transform of derivatives using integration by parts:

$E\left[\frac{\partial f}{\partial t}\right]=\frac{1}{v} T(x, v)-v f(v, 0)$

$E\left[\frac{\partial^{2} f}{\partial t^{2}}\right]=\frac{1}{v^{2}} T(x, v)-f(x, 0)-v \frac{\partial f(x, 0)}{\partial t}$

$E\left[\frac{\partial f}{\partial x}\right]=T^{\prime}(x, v)=\frac{\mathrm{d} T(X, 0)}{\mathrm{d} x}$

$E\left[\frac{\partial f}{\partial x}\right]=T^{\prime \prime}(x, v)=\frac{\mathrm{d}^{2} T(X, 0)}{\mathrm{d} x^{2}}$

Higher-order derivatives with respect to $t$ can be obtained by mathematical induction as:

$E\left[\frac{\partial^{n} f(x . t)}{\partial t^{n}}\right] \Rightarrow \frac{E[f(x, t)]}{v^{n}}-\sum_{k=0}^{n-1} v^{2-n+k} \frac{\partial^{k} f(x, 0)}{\partial t^{k}}$

\section{Homotopy perturbation method}

The homotopy perturbation method was proposed by He [53] who is a Chinese professor of mathematics in 1998; he was able to couple the traditional perturbation method with the homotopy in topology of which he employed in solving some nonlinear breath taking problems.

Homotopy is a fundamental concept in topology and differential geometry [68]. The concept of homotopy can be traced back to rules of Poincare (1854-1982) [69], a French mathematician.

Shortly speaking, a homotopy describes a kind of continuous variation or deformation in mathematics. 
For examples, a circle can be continuously deformed into the square or an ellipse; also, the shape of a coffee cup can be continuously deformed into a doughnut.

However, the shape of a coffee cup cannot be distorted continuously into the shape of a football; essentially, a homotopy defines a connection between different things in mathematics, which contains some characteristics in some aspects [70].

We consider two topological spaces $\left(X, \tau_{1}\right)$ and $\left(Y, \tau_{2}\right)$ of which $f$ and $g$ are continuous maps of the spaces $X$ and $Y, F$ being homotopic to $g$ means a deformation of $f$ into $g$, and if $\left(X, \tau_{1}\right)$ and $\left(Y, \tau_{2}\right)$ are continuous, the $\forall \tau_{1} \in X \exists f^{-1}\left(\tau_{2}\right) \in Y$. (This is surjection.)

It is said that $f$ is homotopic to $g$, if there is a continuous map.

$F: X \times[0,1] \rightarrow Y$

such that

$f(x, 0)=f(x)$ And $f(x, 1)=g(x)$

with the unit interval $[0,1] \forall x \in X$

Then this map is called homotopy between $f$ and $g$

\subsection{Illustration of the method}

For the homotopy perturbation method, we consider a general equation of the type

$D(u)=0$

where $D$ is any differential operator. A convex homotopy (deformation) $H(u, p)$ is defined such that

$H(u, p)=(1-p) F(u)+p D(u)$

$F(u)$ is a fundamental operator with known solution $u_{0}$ which can be obtained easily.

It is clear that $H(u, p)=0$ since $D(u)=0$ and $H(u, p)$ is a convex homotopy on $D(u)$.

For the convex homotopy $H(u, p)$, we have that $H(u, p)=F(u), H(u, 1)=D(u)$.

This monotonic changing process of $p$ from zero to unity $(p=1)$ indicates that the known solution deforms into the original problem $D(u)=0$ where $p \in[0,1]$ is taken as an expanding parameter.

In this method, we first use the imbedding parameter ' $p$ ' as a 'small parameter' and assume that the solution of Eq. (13) can be written as power series in $P$.

$U=u_{0}+p u_{1}+p^{2} u_{2}+p^{3} u_{3}+\cdots+p^{n} u_{n}$

By setting ' $p$ ' to unity $(p \rightarrow 1)$ in Eq. (15), we obtained approximate solution of Eq. (1) to be
$U=\lim _{p \rightarrow 1}(U)=u_{0}+u_{1}+u_{2}+\cdots+u_{n}$

$\therefore U=\lim _{p \rightarrow 1}(U)=\sum_{k=0}^{n} u_{k}$

\section{Application of the Elzaki homotopy transformation perturbation method algorithm to the generalized Burgers- Huxley equation}

We consider the generalized nonlinear Burgers-Huxley equation in (2) subjected to the initial condition $u(x, 0)=f(x)$, and we take the Elzaki transform as:

$E\left\{u_{t}\right\}=E\left\{u_{x x}-\alpha u^{\delta} u_{x}+\beta u\left(1-u^{\delta}\right)\left(u^{\delta}-\gamma\right)\right\}$

Recall that

$E\left\{u_{t}\right\}=\frac{E\{u(x, t)\}}{v}-v u(x, 0)$

$\therefore \frac{E(u(x, t))}{v}-v u(x, 0)=E\left\{u_{x x}-\alpha u^{\delta} u_{x}+\beta u\left(1-u^{\delta}\right)\left(u^{\delta}-\gamma\right)\right\}$

By rearranging terms appropriately and multiplying through by $v$, we have:

$E\{u(x, t)\}=v^{2} u(x, 0)+v E\left\{u_{x x}-\alpha u^{\delta} u_{x}+\beta\left(u\left(1-u^{\delta}\right)\left(u^{\delta}-\gamma\right)\right\}\right.$

By taking the Inverse Elzaki transform of (21), we have:

$$
\begin{aligned}
& E^{-1}[E\{u(x, t)\}]=E^{-1}\left[v^{2} f(x)\right] \\
& \quad+E^{-1}\left[v E\left\{u_{x x}-\alpha u^{\delta} u_{x}+\beta\left(u\left(1-u^{\delta}\right)\left(u^{\delta}-\gamma\right)\right\}\right]\right.
\end{aligned}
$$

$E^{-1}\left[v^{2} f(x)\right]$ gives a new function $G(x, t)$, while $u_{x x}-\alpha u^{\delta} u_{x}+\beta\left(u\left(1-u^{\delta}\right)\left(u^{\delta}-\gamma\right)\right.$ is the arising nonlinear term' of Eq. (2).

$\therefore u(x, t)=G(x, t)+E^{-1}\left[v E\left\{u_{x x}-\alpha u^{\delta} u_{x}+\beta\left(u\left(1-u^{\delta}\right)\left(u^{\delta}-\gamma\right)\right\}\right]\right.$

We now apply the next method in the algorithm; this is the homotopy perturbation method on (23).

Let

$u(x, t)=\sum_{n=0}^{\infty} p^{n} u_{n}(x, t)$

Equation (23) becomes: 
$\sum_{n=0}^{\infty} p^{n} u_{n}(x, t)=G(x, t)+p\left[E^{-1}\left[v E\left\{\begin{array}{c}\left(\sum_{n=0}^{\infty} p^{n} u_{n}\right)_{x x}-\alpha\left(\sum_{n=0}^{\infty} p^{n} u_{n}\right)^{\delta}\left(\sum_{n=0}^{\infty} p^{n} u_{n}\right)_{x} \\ \left.+\beta\left(\sum_{n=0}^{\infty} p^{n} u_{n}\right)\left[1-\left(\sum_{n=0}^{\infty} p^{n} u_{n}\right)^{\delta}\right]\left[\left(\sum_{n=0}^{\infty} p^{n} u_{n}\right)^{\delta}-\gamma\right]\right]\end{array}\right]\right]\right.$

The arising nonlinear terms

$\left(\sum_{n=0}^{\infty} p^{n} u_{n}\right)_{x x}-\alpha\left(\sum_{n=0}^{\infty} p^{n} u_{n}\right)^{\delta}\left(\sum_{n=0}^{\infty} p^{n} u_{n}\right)_{x}+\beta\left(\sum_{n=0}^{\infty} p^{n} u_{n}\right)\left[1-\left(\sum_{n=0}^{\infty} p^{n} u_{n}\right)^{\delta}\right]\left[\left(\sum_{n=0}^{\infty} p^{n} u_{n}\right)^{\delta}-\gamma\right]$

would be decomposed into the a polynomial denoted by

$\sum_{n=0}^{\infty} p^{n} H_{n}(n)$

Then,

$\left[\begin{array}{l}\left(\sum_{n=0}^{\infty} p^{n} u_{n}\right)_{x x}-\alpha\left(\sum_{n=0}^{\infty} p^{n} u_{n}\right)^{\delta}\left(\sum_{n=0}^{\infty} p^{n} u_{n}\right)_{x} \\ \left.+\beta\left(\sum_{n=0}^{\infty} p^{n} u_{n}\right)\left[1-\left(\sum_{n=0}^{\infty} p^{n} u_{n}\right)^{\delta}\right]\left[\left(\sum_{n=0}^{\infty} p^{n} u_{n}\right)^{\delta}-\gamma\right]\right]=\left[\sum_{n=0}^{\infty} p^{n} H_{n}(n)\right]\end{array}\right.$

$\sum_{n=0}^{\infty} p^{n} H_{n}(n)$ is a polynomial in terms of the imbedding parameter $p \in[0,1]$ called the He's polynomial which represents the nonlinear terms. This polynomial can be calculated by the formula

$H_{n}(n)=\frac{1}{n !} \frac{\partial^{n}}{\partial p^{n}} N\left(\sum_{k=0}^{n} p^{k} v_{k}\right)_{p=0}, \quad n=0,1,2, \ldots$

where $N$ is the nonlinear operator.

Then Eq. (25) becomes:

$\sum_{n=0}^{\infty} p^{n} u_{n}(x, t)=G(x, t)+p\left[E^{-1}\left[v E\left[\sum_{n=0}^{\infty} p^{n} H_{n}(u)\right]\right]\right]$

By opening up the series in (28), we obtain:

$p^{0}: u_{0}(x, t)=G(x, t)$

$p^{1}: u_{1}(x, t)=E^{-1}\left[v^{2} E\left[H_{0}(u)\right]\right]$

$p^{2}: u_{2}(x, t)=E^{-1}\left[v^{2} E\left[H_{1}(u)\right]\right]$

$p^{3}: u_{3}(x, t)=E^{-1}\left[v^{2} E\left[H_{2}(u)\right]\right]$

$p^{3}: u_{n}(x, t)=E^{-1}\left[v^{2} E\left[H_{n-1}(u)\right]\right]$

This is the coupling of the Elzaki transform and the homotopy perturbation using the He's polynomial [41, 42] on the Burgers-Huxley equation

Having obtained $u_{0}(x, t), u_{1}(x, t), u_{2}(x, t)$, etc. up to desired iteration, then the solution according to homotopy $(p \rightarrow 1)$ is given by

$$
\begin{aligned}
& p^{0} u_{0}(x, t)+p^{1} u_{1}(x, t)+p^{2} u_{2}(x, t) \\
& \quad+\cdots+p^{n} u_{n}(x, t)=G(x, t)+\left[E^{-1}\left[v E\left\{\begin{array}{c}
p H_{0}(u)+p^{2} H_{1}(u) \\
+p^{3} H_{2}(u)+\cdots+p^{n+1} H_{n}(u)
\end{array}\right\}\right]\right]
\end{aligned}
$$
have

$$
U(x, t)=u_{0}(x, t)+u_{1}(x, t)+u_{2}(x, t)+\cdots+u_{n}(x, t)
$$


Table 1 Case 1

\begin{tabular}{lllll}
\hline & $t$ & EXACT & EHTPM & $\begin{array}{l}\text { ERROR }=\mid \text { EXACT- } \\
\text { EHTPM| }\end{array}$ \\
\hline$x=1$ & 0.1 & 0.6586101677 & 0.6586101685 & 0.0000000008 \\
& 0.2 & 0.6472805375 & 0.6472805381 & 0.0000000006 \\
& 0.3 & 0.6357828413 & 0.6357828418 & 0.0000000005 \\
& 0.4 & 0.6241279918 & 0.6241279926 & 0.0000000008 \\
& 0.5 & 0.6123276001 & 0.6123276008 & 0.0000000007 \\
$x=2$ & 0.1 & 0.7964436586 & 0.7964436604 & 0.0000000018 \\
& 0.2 & 0.7882173830 & 0.7882173813 & 0.0000000017 \\
& 0.3 & 0.7797505961 & 0.7797505954 & 0.0000000007 \\
& 0.4 & 0.7710436411 & 0.7710436401 & 0.0000000001 \\
& 0.5 & 0.7620974978 & 0.7620974995 & 0.0000000017 \\
$x=3$ & 0.1 & 0.8880842585 & 0.8880842583 & 0.0000000002 \\
& 0.2 & 0.8830174664 & 0.8830174663 & 0.0000000001 \\
& 0.3 & 0.8777528603 & 0.8777528605 & 0.0000000002 \\
& 0.4 & 0.8722855969 & 0.8722855969 & 0.0000000000 \\
& 0.5 & 0.8666109785 & 0.8666109781 & 0.0000000004 \\
\hline
\end{tabular}

We present the results for Case 1 at $x=1, x=2, x=3$ for each value of $t=0.1,0.2,0.3,0.4,0.5$ for $\delta=1, \alpha=0, \gamma=1, \beta=1$

Equation (31) results in a Taylor's series of two variables of which its close form can be determined.

\section{Application of algorithm}

\subsection{Example 1 (Case 1)}

In this case, we examine the Burgers-Huxley equation for $\alpha=0, \delta=1, \gamma=1, \beta=1$ given by (Table 1)

$u_{t}=u_{x x}+u(1-u)(u-1)$
Subject to the initial condition

$$
u(x, 0)=\frac{e^{\frac{\sqrt{2}}{4} x}}{e^{\frac{\sqrt{2}}{4} x}+e^{-\frac{\sqrt{2}}{4} x}}
$$

\section{Solution Algorithm}

By taking the Elzaki transform of (32)

$E\left(u_{t}\right)=E\left(u_{x x}\right)+E(u(1-u)(u-1))$

$E\left\{u_{t}\right\}=\frac{E\{u(x, t)\}}{v}-v u(x, 0)$

$E\{u(x, t)\}=v^{2} u(x, 0)+v E\left\{u_{x x}+u(1-u)(u-1)\right\}$

By taking the inverse Elzaki transform of (35)

$$
\begin{aligned}
& E^{-1}[E[u(x, t)]]=E^{-1}\left[v^{2} u(x, 0)\right]+E^{-1}\left[v E\left\{u_{x x}+u(1-u)(u-1)\right\}\right] \\
& \therefore u(x, t)=u(x, 0)+E^{-1}\left[v E\left\{u_{x x}+u(1-u)(u-1)\right\}\right] \\
& \therefore u(x, t)=\frac{\mathrm{e}^{\frac{\sqrt{2}}{4} x}}{\mathrm{e}^{\frac{\sqrt{2}}{4} x}+\mathrm{e}^{-\frac{\sqrt{2}}{4} x}}+E^{-1}\left[v E\left[u_{x x}+u(1-u)(u-1)\right]\right]
\end{aligned}
$$

We now apply the homotopy perturbation method as the next algorithm to Eq. (38).

Let

$u(x, t)=\sum_{n=0}^{\infty} p^{n} u_{n}(x, t)$

By constructing a homotopy on (38), Eq. (38) becomes:

$$
u(x, t)=\frac{\mathrm{e}^{-\frac{x}{4}}}{\mathrm{e}^{\frac{x}{4}}+\mathrm{e}^{-\frac{x}{4}}}+p\left[E^{-1}\left[v E\left[\begin{array}{c}
\left(\sum_{n=0}^{\infty} p^{n} u_{n}\right)_{x x}+\left(\sum_{n=0}^{\infty} p^{n} u_{n}\right)\left(\sum_{n=0}^{\infty} p^{n} u_{n}\right)_{x} \\
+\left(\sum_{n=0}^{\infty} p^{n} u_{n}\right)\left[1-\left(\sum_{n=0}^{\infty} p^{n} u_{n}\right)\right]\left[\left(\sum_{n=0}^{\infty} p^{n} u_{n}\right)-1\right]
\end{array}\right]\right]\right]
$$

The arising nonlinear term in (39) is denoted by $\sum_{n=0}^{\infty} p^{n} H_{n}(n)$

$\therefore\left[\sum_{n=0}^{\infty} p^{n} H_{n}(n)\right]=\left[\begin{array}{l}\left(\sum_{n=0}^{\infty} p^{n} u_{n}(x, t)\right)_{x x}^{\infty} \\ +\left(\sum_{n=0}^{\infty} p^{n} u_{n}(x, t)\right)\left[1-\left(\sum_{n=0}^{\infty} p^{n} u_{n}(x, t)\right)\right]\left[\left(\sum_{n=0}^{\infty} p^{n} u_{n}(x, t)\right)-1\right]\end{array}\right]$ 
By replacing (40) into (39) and inserting initial condition, we have:

$\sum_{n=0}^{\infty} p^{n} u_{n}(x, t)=\frac{\mathrm{e}^{\frac{\sqrt{2}}{4} x}}{\mathrm{e}^{\frac{\sqrt{2}}{4} x}+\mathrm{e}^{-\frac{\sqrt{2}}{4} x}}+p\left[E^{-1}\left[v E\left\{\sum_{n=0}^{\infty} p^{n} H_{n}(u)\right\}\right]\right]$

The first few He's polynomials from the above computed are

$p^{0}: H_{0}(u)=u_{0 x x}-u_{0}^{3}+2 u_{0}^{2}-u_{0}$

$p^{1}: H_{1}(u)=u_{1 x x}-3 u_{0}^{2} u_{1}+4 u_{0} u_{2}^{2}-u_{1}$

$p^{2}: H_{2}(u)=u_{2 x x}-3 u_{0}^{2} u_{2}-3 u_{0} u_{1}^{2}+u_{1}^{2}+4 u_{0} u_{1}-u_{2}$

$p^{3}: H_{3}(u)=u_{3 x x}-u_{1}^{3}-6 u_{0} u_{1} u_{2}+4 u_{1} u_{2}-u_{3}$ $-\frac{1}{2} \frac{1}{\left(e^{\frac{\sqrt{2}}{4} x}+e^{-\frac{\sqrt{2}}{4} x}\right)^{2}}$

is a function of $x$ and would be factored out.

$$
\begin{aligned}
\therefore u_{1}(x, t) & =-\frac{1}{2} \frac{1}{\left(\mathrm{e}^{\frac{\sqrt{2}}{4} x}+\mathrm{e}^{-\frac{\sqrt{2}}{4} x}\right)^{2}} E^{-1}[v E[1]] \\
& =-\frac{1}{2} \frac{1}{\left(\mathrm{e}^{\frac{\sqrt{2}}{4} x}+\mathrm{e}^{-\frac{\sqrt{2}}{4} x}\right)^{2}} E^{-1}\left[v^{3}\right]
\end{aligned}
$$

$\vdots$

$H_{0}(u)=-\frac{1}{2} \frac{1}{\left(e^{\frac{\sqrt{2}}{4} x}+e^{-\frac{\sqrt{2}}{4} x}\right)^{2}} ; H_{1}(u)=-\frac{1}{4} \frac{e^{\frac{\sqrt{2}}{4} x}-e^{-\frac{\sqrt{2}}{4} x}}{\left(e^{\frac{\sqrt{2}}{4} x}+e^{-\frac{\sqrt{2}}{4} x}\right)^{3}} ; H_{2}(u)=-\frac{1}{16} \frac{\left(e^{\frac{\sqrt{2}}{2} x}+e^{-\frac{\sqrt{2}}{2} x}-4\right)}{\left(e^{\frac{\sqrt{2}}{4} x}+e^{-\frac{\sqrt{2}}{4} x}\right)^{4}} t^{2} \cdots$

By comparing the powers of $p$ in Eq. (41), we have

$$
\begin{aligned}
p^{0}: u_{0}(x, t) & =\frac{e^{\frac{\sqrt{2}}{4} x}}{e^{\frac{\sqrt{2}}{4} x}+e^{-\frac{\sqrt{2}}{4} x}} \\
p^{1}: u_{1}(x, t) & =E^{-1}\left[v E\left[H_{0}(u)\right]\right] \\
p^{2}: u_{2}(x, t) & =E^{-1}\left[v E\left[H_{1}(u)\right]\right] \\
p^{3}: u_{3}(x, t) & =E^{-1}\left[v E\left[H_{2}(u)\right]\right] \\
\vdots & \\
p^{n}: u_{n}(x, t) & =E^{-1}\left[v E\left[H_{n-1}(u)\right]\right]
\end{aligned}
$$

Solving (43) accordingly, we obtain the respective solutions of the equation as:

$$
u_{0}(x, t)=u(x, 0)=\frac{\mathrm{e}^{\frac{\sqrt{2}}{4} x}}{\mathrm{e}^{\frac{\sqrt{2}}{4} x}+\mathrm{e}^{-\frac{\sqrt{2}}{4} x}}
$$

From Eq. (43),

$$
u_{1}(x, t)=E^{-1}\left[v E\left[H_{0}(u)\right]\right]=E^{-1}\left[v E\left[-\frac{1}{2} \frac{1}{\left(\mathrm{e}^{\frac{\sqrt{2}}{4} x}+\mathrm{e}^{-\frac{\sqrt{2}}{4} x}\right)^{2}}\right]\right]
$$

$$
\therefore u_{1}(x, t)=-\frac{1}{2} \frac{1}{\left(e^{\frac{\sqrt{2}}{4} x}+e^{-\frac{\sqrt{2}}{4} x}\right)^{2}} t
$$

Similarly,

$$
\begin{aligned}
u_{2}(x, t) & =-\frac{1}{8} \frac{\left(\mathrm{e}^{\frac{\sqrt{2}}{4} x}-\mathrm{e}^{-\frac{\sqrt{2}}{4} x}\right)}{\left(\mathrm{e}^{\frac{\sqrt{2}}{4} x}+\mathrm{e}^{-\frac{\sqrt{2}}{4} x}\right)^{3} t^{2} ; u_{3}(x, t)} \\
& =-\frac{1}{48} \frac{\left(\mathrm{e}^{\frac{\sqrt{2}}{2} x}+\mathrm{e}^{-\frac{\sqrt{2}}{2} x}-4\right)}{\left(\mathrm{e}^{\frac{\sqrt{2}}{4} x}+\mathrm{e}^{-\frac{\sqrt{2}}{4} x}\right)^{4}} t^{3} \cdots
\end{aligned}
$$

Then the solution to the Burgers-Huxley equation according to homotopy from Eq. (31) is given as

$$
\begin{aligned}
u(x, t)= & \frac{\mathrm{e}^{\frac{\sqrt{2}}{4} x}}{\mathrm{e}^{\frac{\sqrt{2}}{4} x}+\mathrm{e}^{-\frac{\sqrt{2}}{4} x}-\frac{1}{2}} \frac{1}{\left(\mathrm{e}^{\frac{\sqrt{2}}{4} x}+\mathrm{e}^{-\frac{\sqrt{2}}{4} x}\right)^{2}} t-\frac{1}{8} \frac{\left(\mathrm{e}^{\frac{\sqrt{2}}{4} x}-\mathrm{e}^{-\frac{\sqrt{2}}{4} x}\right)}{\left(\mathrm{e}^{\frac{\sqrt{2}}{4} x}+\mathrm{e}^{-\frac{\sqrt{2}}{4} x}\right)^{3}} t^{2} \\
& -\frac{1}{48} \frac{\left(\mathrm{e}^{\frac{\sqrt{2}}{2} x}+\mathrm{e}^{-\frac{\sqrt{2}}{2} x}-4\right)}{\left(\mathrm{e}^{\frac{\sqrt{2}}{4} x}+\mathrm{e}^{-\frac{\sqrt{2}}{4} x}\right)^{4} t^{3}+\cdots}
\end{aligned}
$$

Using a computation tool, the above multivariate series solution converges to the closed form. 
Table 2 Case 2

\begin{tabular}{lllll}
\hline & $t$ & EXACT & EHTPM & $\begin{array}{l}\text { ERROR }=\mid \text { EXACT- } \\
\text { EHTPM } \mid\end{array}$ \\
\hline$x=1$ & 0.1 & 0.3600839033 & 0.3600839027 & 0.0000000006 \\
& 0.2 & 0.3429895374 & 0.3429895370 & 0.0000000004 \\
& 0.3 & 0.3262929006 & 0.3262929004 & 0.0000000002 \\
& 0.4 & 0.3100255188 & 0.3100255184 & 0.0000000004 \\
& 0.5 & 0.2942149720 & 0.2942149717 & 0.0000000003 \\
$x=2$ & 0.1 & 0.25445339 & 0.25445339 & 0.00000000 \\
& 0.2 & 0.24048908 & 0.24048907 & 0.00000001 \\
& 0.3 & 0.22705774 & 0.22705772 & 0.00000002 \\
& 0.4 & 0.21416502 & 0.21416445 & 0.00000057 \\
& 0.5 & 0.20181322 & 0.20181132 & 0.00000019 \\
& 0.1 & 0.17150477 & 0.17150477 & 0.00000000 \\
& 0.2 & 0.16110895 & 0.16110894 & 0.00000001 \\
& 0.3 & 0.15122825 & 0.15122814 & 0.00000011 \\
& 0.4 & 0.14185047 & 0.14185049 & 0.00000002 \\
& 0.5 & 0.13296224 & 0.13296217 & 0.00000007 \\
\hline
\end{tabular}

We present the results for Case 2 at $x=1, x=2, x=3$ for each value of $t=0.1,0.2,0.3,0.4,0.5$ for $\delta=1, \alpha=-1, \gamma=1, \beta=1$

$u(x, t)=\frac{1}{2}+\frac{1}{2} \cdot \frac{\mathrm{e}^{\left(\frac{\sqrt{2}}{4} x-\frac{t}{4}\right)}-\mathrm{e}^{-\left(\frac{\sqrt{2}}{4} x-\frac{t}{4}\right)}}{\mathrm{e}^{\left(\frac{\sqrt{2}}{4} x-\frac{t}{4}\right)}+\mathrm{e}^{-\left(\frac{\sqrt{2}}{4} x-\frac{t}{4}\right)}}=\frac{1}{2}+\frac{1}{2} \tanh \left(\frac{\sqrt{2}}{4} x-\frac{t}{4}\right)$

$\therefore u(x, t)=\frac{1}{2}+\frac{1}{2} \tanh \left(\frac{1}{2 \sqrt{2}}\left(x-\frac{t}{\sqrt{2}}\right)\right)$

This corresponds excellently with the general solution

$u_{t}=u_{x x}+u u_{x}+u(1-u)(u-1)$

Subject to the initial condition

$$
u(x, 0)=\frac{\mathrm{e}^{-\frac{x}{4}}}{\mathrm{e}^{\frac{x}{4}}+\mathrm{e}^{-\frac{x}{4}}}
$$

\section{Solution Algorithm}

By taking the Elzaki transform of (49)

$$
\begin{aligned}
& E\left[u_{t}\right]=E\left[u_{x x}\right]+E\left[u u_{x}+u(1-u)(u-1)\right] \\
& E\left\{u_{t}\right\}=\frac{E[u(x, t)]}{v}-v u(x, 0) \\
& E\{u(x, t)\}=v^{2} u(x, 0)+v E\left[u_{x x}+u u_{x}+u(1-u)(u-1)\right]
\end{aligned}
$$

By taking the inverse Elzaki transform of (52)

$$
\begin{aligned}
& E^{-1}[E[u(x, t)]]=E^{-1}\left[v^{2} u(x, 0)\right] \\
& \quad+E^{-1}\left[v E\left\{u_{x x}+u u_{x}+u(1-u)(u-1)\right\}\right] \\
& \therefore u(x, t)=u(x, 0)+E^{-1}\left[v E\left[u_{x x}+u u_{x}+u(1-u)(u-1)\right]\right] \\
& \therefore u(x, t)=\frac{\mathrm{e}^{-\frac{x}{4}}}{\mathrm{e}^{\frac{x}{4}}+\mathrm{e}^{-\frac{x}{4}}}+E^{-1}\left[v E\left[u_{x x}+u u_{x}+u(1-u)(u-1)\right]\right]
\end{aligned}
$$

$u(x, t)=\left[\frac{\gamma}{2}+\frac{\gamma}{2} \tanh \left\{\sigma \gamma\left(x-\left\{\frac{\gamma \alpha}{1+\delta}-\frac{(1+\delta-\gamma)(\rho-\alpha)}{2(1+\delta)}\right\} t\right)\right\}^{1 / \delta}\right.$

obtained by Wang et al. [28] using nonlinear transformations.

where $\sigma=\frac{\delta(\rho-\alpha)}{4(1+\delta)}$ and $\rho=\sqrt{\alpha^{2}+4 \beta(1+\delta)}$ for $\alpha=0, \delta=1, \gamma=1, \beta=1$

\subsection{Example 2 (Case 2)}

Consider the generalized Burgers-Huxley equation in (1) for $\alpha=-1, \delta=1, \gamma=1, \beta=1$ given by (Table 2 )
We now apply the homotopy perturbation method as the next algorithm to Eq. (55)

Let

$u(x, t)=\sum_{n=0}^{\infty} p^{n} u_{n}(x, t)$

By constructing a homotopy on (55), Eq. (55) becomes:

$u(x, t)=\frac{\mathrm{e}^{-\frac{x}{4}}}{\mathrm{e}^{\frac{x}{4}}+\mathrm{e}^{-\frac{x}{4}}}+p\left[E^{-1}\left[v E\left[\begin{array}{r}\left(\sum_{n=0}^{\infty} p^{n} u_{n}\right)_{x x}+\left(\sum_{n=0}^{\infty} p^{n} u_{n}\right)\left(\sum_{n=0}^{\infty} p^{n} u_{n}\right) \\ \left.\left.+\left(\sum_{n=0}^{\infty} p^{n} u_{n}\right)\left[1-\left(\sum_{n=0}^{\infty} p^{n} u_{n}\right)\right]\left[\left(\sum_{n=0}^{\infty} p^{n} u_{n}\right)-1\right]\right]\right]\end{array}\right]\right.\right.$ 
The arising nonlinear term in (56) is denoted by $\sum_{n=0}^{\infty} p^{n} H_{n}(n)$

$\therefore\left[\sum_{n=0}^{\infty} p^{n} H_{n}(n)\right]=\left[\begin{array}{r}\left(\sum_{n=0}^{\infty} p^{n} u_{n}(x, t)\right)_{x x}+\left(\sum_{n=0}^{\infty} p^{n} u_{n}(x, t)\right)\left(\sum_{n=0}^{\infty} p^{n} u_{n}(x, t)\right)_{x} \\ +\left(\sum_{n=0}^{\infty} p^{n} u_{n}(x, t)\right)\left[1-\left(\sum_{n=0}^{\infty} p^{n} u_{n}(x, t)\right)\right]\left[\left(\sum_{n=0}^{\infty} p^{n} u_{n}(x, t)\right)-1\right]\end{array}\right]$

By replacing (57) into (56) and inserting initial condition, we have:

$\sum_{n=0}^{\infty} p^{n} u_{n}(x, t)=\frac{\mathrm{e}^{-\frac{x}{4}}}{\mathrm{e}^{\frac{x}{4}}+\mathrm{e}^{-\frac{x}{4}}}+p\left[E^{-1}\left[v E\left\{\sum_{n=0}^{\infty} p^{n} H_{n}(u)\right\}\right]\right]$

The first few He's polynomials from the above computed are

$$
u_{0}(x, t)=u(x, 0)=\frac{\mathrm{e}^{-\frac{x}{4}}}{\mathrm{e}^{\frac{x}{4}}+\mathrm{e}^{-\frac{x}{4}}}
$$

From Eq. (61),

$$
u_{1}(x, t)=E^{-1}\left[v E\left[H_{0}(u)\right]\right]=E^{-1}\left[v E\left[-\frac{3}{4} \frac{1}{\left(\mathrm{e}^{\frac{x}{4}}+\mathrm{e}^{-\frac{x}{4}}\right)^{2}}\right]\right]
$$

$P^{0}: H_{0}(u)=u_{0 x x}+u_{0} u_{0 x}+2 u_{0}^{2}-u_{0}^{3}-u_{0}$

$p^{1}: H_{1}(u)=u_{1 x x}+u_{0} u_{1 x}+u_{1} u_{0 x}+4 u_{0} u_{1}-3 u_{0}^{2} u_{1}-u_{1}$

$p^{2}: H_{2}(u)=u_{2 x x}+u_{0} u_{2 x}+u_{1} u_{1 x}+u_{2} u_{0 x}+2 u_{1}^{2}+4 u_{0} u_{2}-3 u_{0}^{2} u_{2}-3 u_{0} u_{1}^{2}-u_{2}$

$p^{3}: H_{3}(u)=u_{3 x x}+u_{1} u_{2 x}+u_{2} u_{1 x}+4 u_{1} u_{2}-u_{1}^{3}-6 u_{0} u_{1} u_{2}-u_{3}$

$\vdots$

$H_{0}(u)=-\frac{3}{4} \frac{1}{\left(e^{\frac{x}{4}}+e^{-\frac{x}{4}}\right)^{2}} ;$

$H_{1}(u)=\frac{9}{16} \frac{\left(\mathrm{e}^{\frac{x}{4}}-\mathrm{e}^{-\frac{x}{4}}\right)}{\left(\mathrm{e}^{\frac{x}{4}}+\mathrm{e}^{-\frac{x}{4}}\right)^{3}} t$

$H_{2}(u)=-\frac{27}{128} \frac{\left(\mathrm{e}^{\frac{x}{2}}+\mathrm{e}^{-\frac{x}{2}}-4\right)}{\left(\mathrm{e}^{\frac{x}{2}}+\mathrm{e}^{-\frac{x}{2}}\right)^{4}} t^{2} \cdots$

By comparing the powers of $p$ in Eq. (58), we have

$$
\begin{aligned}
p^{0}: u_{0}(x, t) & =\frac{e^{-\frac{x}{4}}}{e^{\frac{x}{4}}+e^{-\frac{x}{4}}} \\
p^{1}: u_{1}(x, t) & =E^{-1}\left[v E\left[H_{0}(u)\right]\right] \\
p^{2}: u_{2}(x, t) & =E^{-1}\left[v E\left[H_{1}(u)\right]\right] \\
p^{3}: u_{3}(x, t) & =E^{-1}\left[v E\left[H_{2}(u)\right]\right] \\
\vdots & \\
p^{n}: u_{n}(x, t) & =E^{-1}\left[v E\left[H_{n-1}(u)\right]\right]
\end{aligned}
$$

Solving (61) accordingly, we obtain the respective solutions of the equation as: $-\frac{3}{4} \frac{1}{\left(e^{\frac{x}{4}}+e^{-\frac{x}{4}}\right)^{2}}$

is a function of $x$ and would be factored out.

$$
\begin{aligned}
& \therefore u_{1}(x, t)=-\frac{3}{4} \frac{1}{\left(e^{\frac{x}{4}}+e^{-\frac{x}{4}}\right)^{2}} E^{-1}[v E[1]]=-\frac{3}{4} \frac{1}{\left(e^{\frac{x}{4}}+e^{-\frac{x}{4}}\right)^{2}} E^{-1}\left[v^{3}\right] \\
& \therefore u_{1}(x, t)=-\frac{3}{4} \frac{1}{\left(e^{\frac{x}{4}}+e^{-\frac{x}{4}}\right)^{2}} t
\end{aligned}
$$

Similarly,

$$
\begin{aligned}
u_{2}(x, t) & =\frac{9}{32} \frac{\left(\mathrm{e}^{\frac{x}{4}}-\mathrm{e}^{\frac{x}{4}}\right)}{\left(\mathrm{e}^{\frac{x}{4}}+\mathrm{e}^{-\frac{x}{4}}\right)^{3}} t^{2} ; u_{3}(x, t) \\
& =-\frac{9}{128} \frac{\left(\left(\mathrm{e}^{\frac{x}{4}}\right)^{2}+\left(\mathrm{e}^{-\frac{x}{4}}\right)^{2}-4\right)}{\left(\mathrm{e}^{\frac{x}{4}}+\mathrm{e}^{\frac{x}{4}}\right)^{4}} t^{3} \ldots
\end{aligned}
$$

Then the solution to the Burgers-Huxley equation according to homotopy from Eq. (31) is given as 
Table 3 Case 3

\begin{tabular}{lllll}
\hline & $t$ & EXACT & EHTPM & $\begin{array}{l}\text { ERROR }=\mid \text { EXACT- } \\
\text { EHTPM| }\end{array}$ \\
\hline$x=1$ & 0.1 & 0.6539287 & 0.6539287 & 0.0000000 \\
& 0.2 & 0.5668156 & 0.5668156 & 0.0000000 \\
& 0.3 & 0.4888891 & 0.4888891 & 0.0000000 \\
& 0.4 & 0.4198275 & 0.4198272 & 0.0000003 \\
& 0.5 & 0.3591264 & 0.3591261 & 0.0000003 \\
$x=2$ & 0.1 & 0.2551628 & 0.2551628 & 0.0000000 \\
& 0.2 & 0.2162743 & 0.2162730 & 0.0000013 \\
& 0.3 & 0.1829176 & 0.1829124 & 0.0000052 \\
& 0.4 & 0.1544203 & 0.1544233 & 0.0000003 \\
& 0.5 & 0.1301576 & 0.1301578 & 0.0000002 \\
& 0.1 & 0.0902139 & 0.0902139 & 0.0000000 \\
& 0.2 & 0.0757707 & 0.0757704 & 0.0000003 \\
& 0.3 & 0.0635844 & 0.0635842 & 0.0000002 \\
& 0.4 & 0.0533306 & 0.0533304 & 0.0000002 \\
& 0.5 & 0.0447017 & 0.0447014 & 0.0000003 \\
\hline
\end{tabular}

We present the results for Case 3 at $x=1, x=2, x=3$ for each value of $t=0.1,0.2,0.3,0.4,0.5$ for $\delta=1, \alpha=-2, \gamma=3, \beta=1$ obtained by Wang et al. [28] using nonlinear transformations.

where $\sigma=\frac{\delta(\rho-\alpha)}{4(1+\delta)}$ and $\rho=\sqrt{\alpha^{2}+4 \beta(1+\delta)}$ for $\alpha=-1, \delta=1, \gamma=1, \beta=1$

\subsection{Example 3 (Case 3)}

Consider the generalized Burgers-Huxley equation in (1) for $\delta=1, \alpha=-2, \gamma=3, \beta=1$ given by (Table 3)

$u_{t}=u_{x x}+u u_{x}+2 u(1-u)(u-3)$

Subject to the initial condition

$u(x, 0)=\frac{3 e^{-\frac{-3(\sqrt{3}-1)}{4} x}}{e^{\frac{3(\sqrt{3}-1)}{4} x}+e^{\frac{-3(\sqrt{3}-1)}{4} x}}$

\section{Solution Algorithm}

By taking the Elzaki transform of (67)

$E\left(u_{t}\right)=E\left(u_{x x}\right)+E\left[u_{x x}+u u_{x}+2 u(1-u)(u-3)\right]$
Using a computation tool, the above multivariate series solution converges to the closed form.

$$
\begin{aligned}
& u(x, t)=\frac{1}{2}-\frac{1}{2} \frac{\mathrm{e}^{\left(\frac{2 x}{8}+\frac{3 t}{8}\right)}-\mathrm{e}^{-\left(\frac{2 x}{8}+\frac{3 t}{8}\right)}}{\mathrm{e}^{\left(\frac{2 x}{8}+\frac{3 t}{8}\right)}+\mathrm{e}^{-\left(\frac{2 x}{8}+\frac{3 t}{8}\right)}}=\frac{1}{2}-\frac{1}{2} \tanh \left[\frac{1}{4}\left(x+\frac{3 t}{2}\right)\right] \\
& \therefore u(x, t)=\frac{1}{2}-\frac{1}{2} \tanh \left[\frac{1}{4}\left(x+\frac{3 t}{2}\right)\right]
\end{aligned}
$$

This corresponds excellently with the general solution

$$
E\left\{u_{t}\right\}=\frac{E\{u(x, t)\}}{v}-v u(x, 0)
$$

$\therefore E\{u(x, t)\}=v^{2} u(x, 0)+v E\left[u_{x x}+u u_{x}+2 u(1-u)(u-3)\right]$

By taking the inverse Elzaki transform of (70)

$$
\begin{aligned}
& E^{-1}[E[u(x, t)]]=E^{-1}\left[v^{2} u(x, 0)\right] \\
& \quad+E^{-1}\left[v E\left[u_{x x}+u u_{x}+2 u(1-u)(u-3)\right]\right]
\end{aligned}
$$

$u(x, t)=\left[\frac{\gamma}{2}+\frac{\gamma}{2} \tanh \left\{\sigma \gamma\left(x-\left\{\frac{\gamma \alpha}{1+\delta}-\frac{(1+\delta-\gamma)(\rho-\alpha)}{2(1+\delta)}\right\} t\right)\right\}\right]^{1 / \delta}$

$$
\therefore u(x, t)=u(x, 0)+E^{-1}\left[v E\left\{u_{x x}+u u_{x}+2 u(1-u)(u-3)\right\}\right]
$$

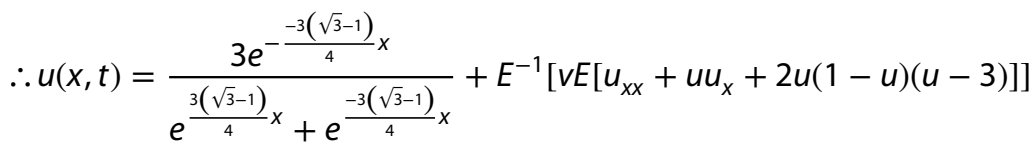


We now apply the homotopy perturbation method as the next algorithm to Eq. (72)

Let

$u(x, t)=\sum_{n=0}^{\infty} p^{n} u_{n}(x, t)$

$$
H_{2}(u)=-\frac{81}{16} \frac{\left(e^{\frac{3(\sqrt{3}-1)}{2} x}+e^{-\frac{3(\sqrt{3}-1)}{2} x}-4\right)(389-225 \sqrt{3})}{\left(e^{\frac{3(\sqrt{3}-1)}{4} x}+e^{-\frac{3(\sqrt{3}-1)}{4} x}\right)^{4}} t^{2} \cdots
$$

By comparing the powers of $p$ in Eq. (41), we have

By constructing a homotopy on (73) and replacing $u(x, t)$, Eq. (73) becomes:

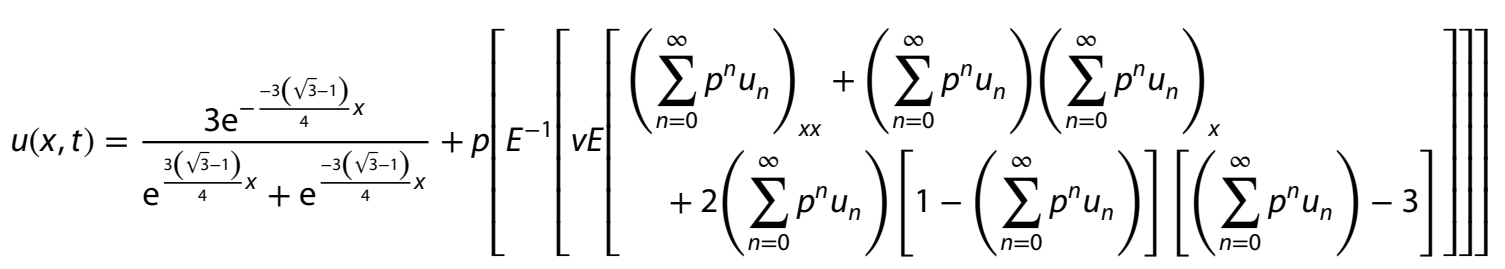

The arising nonlinear terms in (74) would be denoted

by $\sum_{n=0}^{\infty} p^{n} H_{n}(n)$

$\therefore\left[\sum_{n=0}^{\infty} p^{n} H_{n}(n)\right]=\left[\begin{array}{l}\left(\sum_{n=0}^{\infty} p^{n} u_{n}(x, t)\right)_{x x}+\left(\sum_{n=0}^{\infty} p^{n} u_{n}(x, t)\right)\left(\sum_{n=0}^{\infty} p^{n} u_{n}(x, t)\right)_{x} \\ \quad+2\left(\sum_{n=0}^{\infty} p^{n} u_{n}(x, t)\right)\left[1-\left(\sum_{n=0}^{\infty} p^{n} u_{n}(x, t)\right)\right]\left[\left(\sum_{n=0}^{\infty} p^{n} u_{n}(x, t)\right)-3\right]\end{array}\right]$

By replacing (75) into (74) and inserting initial condi-

tion, we have:

$\sum_{n=0}^{\infty} p^{n} u_{n}(x, t)=\frac{3 \mathrm{e}^{-\frac{-3(\sqrt{3}-1)}{4} x}}{\mathrm{e}^{\frac{3(\sqrt{3}-1)}{4} x}+\mathrm{e}^{\frac{-3(\sqrt{3}-1)}{4} x}}+p\left[E^{-1}\left[v E\left\{\sum_{n=0}^{\infty} p^{n} H_{n}(u)\right\}\right]\right]$

The first few He's polynomials from the above com-

puted are

$P^{0}: H_{0}(u)=u_{0 x x}+2 u_{0} u_{0 x}+4 u_{0}^{2}-u_{0}^{3}-3 u_{0}$

$p^{1}: H_{1}(u)=u_{1 x x}+2 u_{0} u_{1 x}+2 u_{1} u_{0 x}+8 u_{0} u_{1}-3 u_{0}^{2} u_{1}-3 u_{1}$

$p^{2}: H_{2}(u)=u_{2 x x}+2 u_{0} u_{2 x}+2 u_{1} u_{1 x}+2 u_{2} u_{0 x}+4 u_{1}^{2}+8 u_{0} u_{2}-3 u_{0}^{2} u_{2}-3 u_{0} u_{1}^{2}-3 u_{2}$

$p^{3}: H_{3}(u)=u_{3 x x}+2 u_{1} u_{2 x}+2 u_{2} u_{1 x}+8 u_{1} u_{2}-u_{1}^{3}-6 u_{0} u_{1} u_{2}-3 u_{3}$

$\vdots$

$H_{0}(u)=\frac{-9}{2} \frac{(3 \sqrt{3}-4)}{\left(\mathrm{e}^{\frac{3(\sqrt{3}-1)}{4} x}+\mathrm{e}^{-\frac{3(\sqrt{3}-1)}{4} x}\right)^{2}} ; H_{1}(u)=\frac{27}{16} \frac{\left(\mathrm{e}^{\frac{3(\sqrt{3}-1)}{4} x}-\mathrm{e}^{-\frac{3(\sqrt{3}-1)}{4} x}\right)(43-24 \sqrt{3})}{\left(\mathrm{e}^{\frac{3(\sqrt{3}-1)}{4} x}+\mathrm{e}^{-\frac{3(\sqrt{3}-1)}{4} x}\right)^{3}} t ;$ 
$p^{0}: u_{0}(x, t)=\frac{3 e^{-\frac{-3(\sqrt{3}-1)}{4} x}}{\mathrm{e}^{\frac{3(\sqrt{3}-1)}{4} x}+\mathrm{e}^{\frac{-3(\sqrt{3}-1)}{4} x}}$

$p^{1}: u_{1}(x, t)=E^{-1}\left[v E\left[H_{0}(u)\right]\right]$

$p^{2}: u_{2}(x, t)=E^{-1}\left[v E\left[H_{1}(u)\right]\right]$

$p^{3}: u_{3}(x, t)=E^{-1}\left[v E\left[H_{2}(u)\right]\right]$

$\vdots$

$p^{n}: u_{n}(x, t)=E^{-1}\left[v E\left[H_{n-1}(u)\right]\right]$

Solving (78) accordingly, we obtain the respective solutions of the equation as:

$u_{0}(x, t)=u(x, 0)=\frac{-9}{2} \frac{(3 \sqrt{3}-4)}{\left(\mathrm{e}^{\frac{3(\sqrt{3}-1)}{4} x}+\mathrm{e}^{-\frac{3(\sqrt{3}-1)}{4} x}\right)^{2}}$

From Eq. (78),

$u_{1}(x, t)=E^{-1}\left[v E\left[H_{0}(u)\right]\right]=E^{-1}\left[v E\left[\frac{-9}{2} \frac{(3 \sqrt{3}-4)}{\left(\mathrm{e}^{\frac{3(\sqrt{3}-1)}{4} x}+\mathrm{e}^{-\frac{3(\sqrt{3}-1)}{4} x}\right)^{2}}\right]\right]$
Similarly,

$u_{2}(x, t)=\frac{27}{16} \frac{\left(\mathrm{e}^{\frac{3(\sqrt{3}-1)}{4} x}-\mathrm{e}^{-\frac{3(\sqrt{3}-1)}{4} x}\right)(43-24 \sqrt{3})}{\left(\mathrm{e}^{\frac{3(\sqrt{3}-1)}{4} x}+\mathrm{e}^{-\frac{3(\sqrt{3}-1)}{4} x}\right)^{3}} t^{2}$

$$
=\frac{-9}{2} \frac{(3 \sqrt{3}-4)}{\left(\mathrm{e}^{\frac{3(\sqrt{3}-1)}{4} x}+\mathrm{e}^{-\frac{3(\sqrt{3}-1)}{4} x}\right)^{2}} E^{-1}\left[v^{3}\right]
$$

$\therefore u_{1}(x, t)=\frac{-9}{2} \frac{(3 \sqrt{3}-4)}{\left(\mathrm{e}^{\frac{3(\sqrt{3}-1)}{4} x}+\mathrm{e}^{-\frac{3(\sqrt{3}-1)}{4} x}\right)^{2}} t$

Then the solution to the Burgers-Huxley equation according to homotopy from Eq. (31) is given as

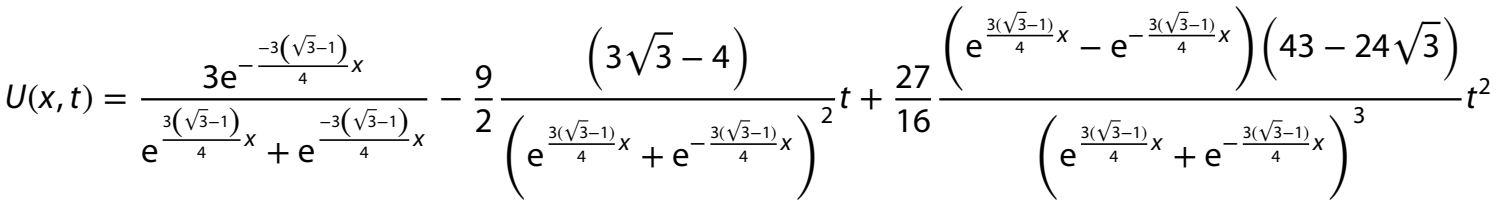

$$
\begin{aligned}
& -\frac{81}{32}\left(\frac{1}{\left(\mathrm{e}^{\frac{3(\sqrt{3}-1)}{4} x}+\mathrm{e}^{-\frac{3(\sqrt{3}-1)}{4} x}\right)^{4}}\right)\left(\mathrm{e}^{\frac{3(\sqrt{3}-1)}{2} x}-\mathrm{e}^{-\frac{3(\sqrt{3}-1)}{2} x}-4\right)(389-225 \sqrt{3}) t^{3}+\cdots
\end{aligned}
$$

Using a computation tool, the above multivariate series solution converges to the closed form. 
$u(x, t)=\frac{3}{2}-\frac{3}{2} \frac{e^{\frac{3 \sqrt{3}-3}{4} x+\frac{9 \sqrt{3}-12}{4} t}-\mathrm{e}^{-\frac{3 \sqrt{3}-3}{4} x-\frac{9 \sqrt{3}-12}{4} t}}{\mathrm{e}^{\frac{3 \sqrt{3}-3}{4} x+\frac{9 \sqrt{3}-12}{4} t}+\mathrm{e}^{-\frac{3 \sqrt{3}-3}{4} x-\frac{9 \sqrt{3}-12}{4} t}}=\frac{3}{2}-\frac{3}{2} \tanh \left[\frac{3 \sqrt{3}-3}{4}\left(x+\frac{5-\sqrt{3}}{2} t\right)\right]$

$\therefore u(x, t)=\frac{3}{2}-\frac{3}{2} \tanh \left[\frac{3 \sqrt{3}-3}{4}\left(x+\frac{5-\sqrt{3}}{2} t\right)\right]$

This corresponds excellently with the general solution
The EHTPM results when compared to the exact obtained from the prominent literatures and to using the ordinary homotopy $[28,63]$ clearly show a high level of rapid convergence and exactness than the ordinary homotopy. These solutions obtained via the proposed

$u(x, t)=\left[\frac{\gamma}{2}+\frac{\gamma}{2} \tanh \left\{\sigma \gamma\left(x-\left\{\frac{\gamma \alpha}{1+\delta}-\frac{(1+\delta-\gamma)(\rho-\alpha)}{2(1+\delta)}\right\} t\right)\right\}\right]^{1 / \delta}$

obtained by Wang et al. [28] using nonlinear transformations.

where $\sigma=\frac{\delta(\rho-\alpha)}{4(1+\delta)}$ and $\rho=\sqrt{\alpha^{2}+4 \beta(1+\delta)}$ for $\delta=1, \alpha=-2, \gamma=3, \beta=1$

\section{Results}

In this section, we present a relationship and comparison between the exact results and the Elzaki homotopy transformation perturbation (EHTPM) results for the three cases of the Burgers-Huxley equation using tables and 3D-Plots.

The exact results were obtained from a general solution presented by Wang et al. [28] as:
EHTPM are easily interpreted as it is presented in a Taylor multivariate series and closed form with a precise and straightforward algorithm, with no restrictions, discretization and devoid of errors.

On this note, we strongly recommend the proposed EHTPM in solving models in fluid flow and fluid dynamics, engineering, nonlinear dynamics, acoustics, convection diffusion models, advection diffusion models and so on. In addition, this method is highly efficient enough and can be brought into the classroom to provide analytical solutions to equations similar to the Burgers-Huxley equation and other nonlinear partial differential equation as well.

$u(x, t)=\left[\frac{\gamma}{2}+\frac{\gamma}{2} \tanh \left\{\sigma \gamma\left(x-\left\{\frac{\gamma \alpha}{1+\delta}-\frac{(1+\delta-\gamma)(\rho-\alpha)}{2(1+\delta)}\right\} t\right)\right\}\right]^{1 / \delta}$

where $\sigma=\frac{\delta(\rho-\alpha)}{4(1+\delta)}$, and $\rho=\sqrt{\alpha^{2}+4 \beta(1+\delta)}$

\section{Conclusion}

We have studied the Burgers-Huxley equation of three cases using the Elzaki homotopy transformation perturbation method (EHTPM). This method is unprecedented.
Acknowledgements The authors of this research article express their profound gratitude and sincerest thanks to the anonymous reviewers, for their constructive opinions towards making this research article scientifically sound.

\section{Compliance with ethical standards}

Conflict of interest The authors of this research have declared no conflict of interest relevant to this article. 


\section{Appendix}

\section{Elzaki table of transform for some functions}

\begin{tabular}{ll}
\hline$f(t)$ & $E[f(t)]=T(v)$ \\
1 & $v^{2}$ \\
$t$ & $v^{3}$ \\
$t^{n}$ & $n ! v^{n+2}$ \\
$\mathrm{e}^{a t}$ & $\frac{v^{2}}{1-a v}$ \\
$t \mathrm{e}^{a t}$ & $\frac{v^{3}}{(1-a v)^{2}}$ \\
$\sin a t$ & $\frac{a v^{3}}{1+a^{2} v^{2}}$ \\
$\cos a t$ & $\frac{u^{2}}{1+a^{2} v^{2}}$ \\
$\sinh a t$ & $\frac{a v^{3}}{1-a^{2} v^{2}}$ \\
$\cosh a t$ & $\frac{a v^{2}}{1-a^{2} v^{2}}$ \\
$\mathrm{e}^{a t} \sin b t$ & $\frac{b v^{3}}{(1-a v)^{2}+b^{2} v^{2}}$ \\
$\mathrm{e}^{a t} \cos b t$ & $\frac{(1-a v) v^{2}}{(1-a v)^{2}+b^{2} v^{2}}$ \\
$t \sin a t$ & $\frac{2 a v^{4}}{1+a^{2} v^{2}}$ \\
$t^{a-1} / \Gamma(a), a=0$ & $v^{a+1}$ \\
$\frac{t^{n-1} \mathrm{e}^{a t}}{(n-1) !}, n=1,2, \ldots$ & $\frac{v^{n+1}}{(1-a v)^{n}}$ \\
$J_{0}(a t)$ & $\frac{v^{2}}{\sqrt{1+a v^{2}}}$ \\
$H(t-a)$ & $v^{2} \mathrm{e}^{-\frac{a}{v}}$ \\
$\delta(t-a)$ & $\frac{v \mathrm{e}^{-\frac{a}{v}}}{}$ \\
\hline &
\end{tabular}

\section{References}

1. Zulkiflee FB (2017) Elzaki transform homotopy perturbation method for partial differential equations. Master thesis, Universiti Teknologi Malaysia

2. Shaeer MJAR (2013) Solutions to nonlinear partial differential equations by Tan-Cot method. IOSR J Math (IOSR-JM) 5(3):6-11

3. Salas $A H$ (2012) Solving nonlinear partial differential equations by the sn-ns method. Abstr Appl Anal Article ID 340824. https ://doi.org/10.1155/2012/340824

4. Dhunde RR, Waghmare GL (2019) Double Laplace iterative method for solving nonlinear partial differential equations. New Trends Math Sci 7(2):138-149

5. Kumbinarasaiah S (2019) Numerical solution of partial differential equations using Laguerre wavelets collocation method. Int J Manag Technol Eng 9(1):3635-3639

6. Aziz I, Siraj-ul-Islam I, Asif M (2017) Haar wavelet collocation method for three-dimensional elliptic partial differential equations. Comput Math Appl. https://doi.org/10.1016/j.camwa .2017.02.034

7. He JH (1999) Variational iteration method-a kind of non-linear analytical technique: some examples. Int J Non Linear Mech 34(4):699-708. https://doi.org/10.1016/S0020-7462(98)00048-1

8. Loyinmi AC, Lawal OW, Sottin DO (2017) Reduced differential transform method for solving partial integro-differential equation. J Nigerian Assoc Math Phys 43:37-42

9. Hashim I, Noorani MSM, Said Al-Hadidi MR (2006) Solving the generalized Burgers-Huxley equation using the Adomian decomposition method. Math Comput Model 43:1404-1411. https://doi.org/10.1016/j.mcm.2005.08.017
10. Bilidik N (2017) General convergence analysis for the perturbation iteration technique. Turk J Math Comput Sci 6:1-9

11. List of Nonlinear partial differential equations, Wkipedia. https ://en.wikipedia.org/wiki/List_of_nonlinear_partial_differenti al_equations

12. Wazwaz A-M (2009) Partial differential equations and solitary waves theory. Springer, Berlin

13. Deniz S, Bildik N (2017) A note on stability analysis of Taylor collocation method. Celal Bayar Üniversitesi Fen Bilimleri Dergisi. https://doi.org/10.18466/cbayarfbe.302660

14. Sinan D (2013) Applications of Taylor collocation method and Lambert $W$ function to the systems of delay differential equations. Turk J Math Comput Sci 20130033:13

15. Bildik N, Tosun M, Deniz S (2017) Euler matrix method for solving complex differential equations with variable coefficients in rectangular domains. Int J Appl Phys Math 7(1):69-78. https:// doi.org/10.17706/ijapm.2017.7.1.69-78

16. Mehra M Applications of Wavelets to partial differential equations. Department of Mathematics, Mc Master University, Canada. http://web.iitd.ac.in/ mmehra/TIFR_talk.pdf

17. Tomasiello $S$ (2010) Numerical solutions of the Burgers-Huxley equation by the IDQ method. Int J Comput Math 87(1):129-140. https://doi.org/10.1080/00207160801968762

18. He JH (2000) variational iteration method for autonomous ordinary differential systems. Appl Math Comput 114(2-3):115-123. https://doi.org/10.1016/S0096-3003(99)00104-6

19. Soori $M(2016$, November 21) The variational iteration method for the Newell-Whitehead-Segel equation. Zenodo. http://doi. org/10.5281/zenodo. 167857

20. He J-H (2003) Homotopy perturbation method: a new nonlinear analytical technique. Appl Math Comput AMC 135:73-79. https ://doi.org/10.1016/s0096-3003(01)00312-5

21. He J-H (2005) Application of homotopy perturbation method to nonlinear wave Equations. Chaos Solitons Fractals 26:695-700. https://doi.org/10.1016/j.chaos.2005.03.006

22. He J-H (2006) Homotopy perturbation method for solving boundary value problems. Phys Lett A 350(2):87-88. https:// doi.org/10.1016/j.physleta.2005.10.005

23. Mountassir Hamdi Cherif, Belghaba Kacem, Ziane Djelloul (2016) Homotopy perturbation method for solving the fractional Fisher's equation. J Anal Appl 10:9-16

24. Mt Senol, Kasmaei HD (2017) Perturbation-iteration algorithm for systems of fractional differential equations and convergence analysis. Prog Fract Differ Appl 3:271-279. https://doi. org/10.18576/pfda/030403

25. Loyinmi AC, Erinle-Ibrahim LM, Adeyemi AE (2017) The new iterative method for solving telegraph equation. J Niger Assoc Math Phys 43:31-36

26. Lu D, Liu J (2014) Application of the homotopy analysis method for solving the variable coefficient KdV-Burgers equation. Abstr Appl Anal 2014, Article ID 309420. https://doi. org/10.1155/2014/309420

27. Abdulaziz O, Hashim I, Saif A (2008) Series solutions of time-fractional PDEs by homotopy analysis method. Differ Equ Nonlinear Mech 2008, Article ID 686512. https://doi. org/10.1155/2008/686512

28. Wang $X Y$ (1985) Nerve propagation and wall in liquid crystals. Phys Lett A 112:402-406. https://doi.org/10.1016/03759601(85)90411-6

29. El-Danaf T (2011) Solitary wave solutions for the generalized Burgers-Huxley equation. Int J Nonlinear Sci Numer Simul 8(3):315-318. https://doi.org/10.1515/ijnsns.2007.8.3.315

30. Ma W-X, Zhou Y (2016) Lump solutions to nonlinear partial differential equations via Hirota bilinear forms. J Differ Equ 264(4):2633-2659. https://doi.org/10.1016/j.jde.2017 
31. Liu Y, Wen X-Y (2019) Soliton, breather, lump and their interaction solutions of the $(2+1)$-dimensional asymmetrical NizhnikNovikov-Veselov equation. Adv Differ Equ 2019:332. https://doi. org/10.1186/s13662-019-2271-5

32. Priyanka C, Karthikeyan N (2017) Solving nonlinear partial differential equations by using Sumudu decomposition method. Int J Eng Dev Res 6(3):589-591

33. Bildik N, Deniz S (2016) The use of Sumudu decomposition method for solving predator-prey systems. Math Sci Lett 5:285289. https://doi.org/10.18576/msl/050310

34. Ramadan MA-L, Al-luhaibi MS (2016) Application of Sumudu decomposition method for solving nonlinear wave-like equations with variable coefficients. Electron J Math Anal Appl 4(1):116-124

35. Tripathi R, Mishra HK (2016) Homotopy perturbation method with Laplace transform (LT-HPM) for solving Lane-Emden type differential equations (LETDEs). Springer Plus 5:1859. https:// doi.org/10.1186/s40064-016-3487-4

36. Johnston S, Jafari H, Moshokoa S et al (2016) Laplace homotopy perturbation method for Burgers equation with space- and time-fractional order. Open Phys 14(1):247-252. https://doi. org/10.1515/phys-2016-0023

37. Jin $L$ (2009) Application of variational iteration method and homotopy perturbation to the modified Kawahara equation. Math Comput Model 49(3-4):573-578. https://doi.org/10.1016/j. $\mathrm{mcm} .2008 .06 .017$

38. Elzaki TM (2012) Solution to nonlinear differential equations using mixture of Elzaki transform and differential transform method. Int Math Forum 7(13):631-638

39. Khalouta A, Kademi A (2018) Mixed of Elzaki transform and projected differential transform method for a nonlinear wave-like equations with variable coefficients. Preprints 2018. https://doi. org/10.20944/preprints201808.0088.v1

40. T Elzaki (2014) Projected differential transform method and Elzaki transform for solving system of nonlinear partial differential equations. World Appl Sci J 32(9):1974-1979. https://doi. org/10.5829/idosi.wasj.2014.32.09.1253

41. Elzaki T, Hilal EMA (2012) Homotopy perturbation and Elzaki transform for solving nonlinear partial differential equations. Math Theory Model 2(3):33-42

42. Elzaki Tarig, Biazar J (2013) Homotopy perturbation and Elzaki transform for solving system of nonlinear partial differential equations. World Appl Sci J 24(7):944-948

43. Jena RM, Chakraverty S (2018) Solving the time fractional Navier-Stokes equations using homotopy perturbation Elzaki transform. SN Appl Sci 1:16. https://doi.org/10.1007/s4245 2-018-0016-9

44. Majid Khan M, Hussain Hossein Jafari, Khan Yasir (2013) Application of laplace decomposition method to solve nonlinear coupled partial differential equations. World Appl Sci J 9:13-19

45. Elzaki TM (2018) Solution to nonlinear partial differentials by new Laplace variational iteration method. IntechOpen 9:153171. https://doi.org/10.5772/intechopen.7329

46. Elzaki TM, Elnour EA (2013) Solution of nonlinear partial differential equations by the combined Laplace transform and the new modified variational iteration method. Wulfenia J 20(4):174-180

47. Nadeem M, Li F, Ahmad H (2019) Modified Laplace variational iteration method for solving fourth-order parabolic partial differential equation with variable coefficients. Comput Math Appl. https://doi.org/10.1016/j.camwa.2019.03.053

48. Cherif MH, Ziane D (2018) Variational iteration method combined with new transform to solve fractional partial differential equations. Univers J Math Appl 1(2):113-120. https://doi. org/10.32323/ujma.396941

49. Elzaki Tarig (2011) New integral transform "Elzaki transform". Glob J Pure Appl Math 7(1):57-64
50. Tarig Elzaki, Salih Ezaki (2011) Application of new transform "Elzaki transform" to partial differential equations. Glob J Pure Appl Math 7(1):65-70

51. Elzaki T, Ezaki S (2011) On the Elzaki transform and ordinary differential equation with variable coefficients. Adv Theor Appl Math 6(1):41-46

52. Elzaki T, Ezaki S, Elnour EA (2012) Application of new transform "Elzaki transform" to mechanics, electrical circuits and beams. Glob J Pure Appl Math 4(1):25-34

53. He JH (1999) Homotopy perturbation technique. Comput Methods Appl Mech Eng 178:257-262. https://doi.org/10.1016/S0045 -7825(99)00018-3

54. Weerakoon S (1994) Application of Sumudu transform to partial differential equations. Int J Math Educ Sci Technol 25(2):277283. https://doi.org/10.1080/0020739940250214

55. Kaya F, Yilmaz Y (2019) Basic properties of Sumudu transform and its application to some partial differential equations. Sakarya Univ J Sci 23(4):509-514. https://doi.org/10.16984/saufe nbilder.416501

56. Satsuma J (1987) Topics in soliton theory and exactly solvable nonlinear equations. World Scientific, Singapore

57. Bateman H (1915) Some recent researches on the motion of fluids. Mon Weather Rev 43(4):163-170

58. Whitham GB (2011) Linear and nonlinear waves. Wiley, New York, p 42

59. Burgers JM (1948) A mathematical model illustrating the theory of turbulence. Adv Appl Mech 1:171-199

60. Hodgkin AL, Huxley AF (1952) A quantitative description of membrane current and its application to conduction and excitation in nerve. J Physiol 117(4):500-544. https://doi.org/10.1113/ jphysiol.1952.sp004764

61. Freihat Asad, Zurigat Mohammad (2019) Analytical solution of fractional Burgers-Huxley Equations via residual power series method. Lobachevskii J Math 40:174-182

62. Zhu MX (2016) Solving the Burgers-Huxley equation by $G^{\prime} / G$ expansion method. J Appl Math Phys 4:1371-1377. https://doi. org/10.4236/jamp.2016.47146

63. Nourazar SS, Soori M, Nazari-Golshan A (2015) On the exact solution of Burgers-Huxley equation using the homotopy perturbation method. J Appl Math Phys 3:285-294. https://doi. org/10.4236/jamp.2015.33042

64. Mittal RC, Tripathi A (2015) Numerical solutions of generalized Burgers-Fisher and generalized Burgers-Huxley equations using collocation of cubic B-splines. Int J Comput Math 92(5):1053-1077. https://doi.org/10.1080/00207160.2014.920834

65. Kamboj D, Sharma MD (2013) Singularly perturbed Burger-Huxley equation: analytical solution through iteration. Int J Eng Sci Tecnol 5(3):45-57

66. Feng Zhaosheng, Tian Jing, Zheng Shenzhou, Lu Hanfang (2012) Travelling wave solutions of the Burgers-Huxley equation. IMA J Appl Math 77:316-325

67. Deng XJ (2008) Travelling wave solutions for the generalized Burgers-Huxley equation. Appl Math Comput 204:733-737. https://doi.org/10.1016/j.amc.2008.07.020

68. Armstrong MA (1983) Basic topology (undergraduate texts in mathematics). Springer, New York

69. Poincare H (1886) Sur les Integrals Irreguliueres. Acta Math 8

70. Ahmed MES (2016) Application of homotopy perturbation method to linear and nonlinear partial differential equations. $\mathrm{PhD}$ thesis, Sudan University of Science and Technology, College of Graduate Studies

Publisher's Note Springer Nature remains neutral with regard to jurisdictional claims in published maps and institutional affiliations. 